\title{
Stability and reliability of plasma level of lipid biomarkers and their correlation with dietary fat intake
}

\author{
Sang-Ah Lee ${ }^{\mathrm{a}}$, Wanqing Wen ${ }^{\mathrm{a}}$, Yong-Bing Xiang ${ }^{\mathrm{b}}$, Sergio Fazio ${ }^{\mathrm{a}, \mathrm{c}}$, MacRae F. Linton ${ }^{\mathrm{a}, \mathrm{c}}$, Qiuyin Cai ${ }^{\mathrm{a}}$, \\ Dake Liu $^{\mathrm{b}}$, Wei Zheng ${ }^{\mathrm{a}}$ and Xiao-Ou Shu ${ }^{\mathrm{a}, *}$ \\ ${ }^{a}$ Department of Medicine, Vanderbilt Epidemiology Center, Vanderbilt University Medical Center and Vanderbilt \\ Ingram Cancer Center, Nashville, TN, USA \\ ${ }^{\mathrm{b}}$ Department of Epidemiology, Shanghai Cancer Institute, Shanghai, P.R. China \\ ${ }^{\mathrm{c}}$ Department of Pathology, Vanderbilt University Medical Center, Nashville, TN, USA
}

\begin{abstract}
The reliability and stability of plasma lipid biomarkers and their association with dietary fat intake were evaluated among 48 subjects who were randomly chosen from the participants of a validation study of the population-based cohort, the Shanghai Men's Health Study (SMHS). Four spot blood samples, one taken each season, were measured for total cholesterol, triglyceride, HDL-cholesterol, and LDL-cholesterol levels. The reliability and stability of these measurements were assessed by intraclass correlation coefficients (ICC) and by the correlations between a randomly chosen measurement with the mean of measurements across seasons using a bootstrap approach. The median levels for total cholesterol, triglycerides, HDLcholesterol, and LDL-cholesterol were 177.5, 164.5, 41.0, and 102.5 (mg/dl), respectively. The ICCs of the biomarkers ranged from 0.58 (LDL-cholesterol) to 0.83 (HDL-cholesterol). The correlation between randomly chosen spot measurements and the mean measurement were $0.91,0.86,0.93$, and 0.83 for total cholesterol, triglycerides, HDL-cholesterol, and LDL-cholesterol, respectively. The correlations of lipid biomarkers with dietary fat intake and other lifestyle factors were comparable to other previous reports. In conclusion, this study suggests that measurements of lipid biomarkers from a single spot blood sample are a good representation of the average blood levels of these biomarkers in the study population and could be a useful tool for epidemiological studies.
\end{abstract}

\section{Introduction}

Morbidity and mortality from cardiovascular disease (CVD), type 2 diabetes mellitus, and hypertension, important components of the metabolic syndrome, are expected to dramatically increase during the next 20 years in China and other developing countries [1]. CVD accounted for nearly $40 \%$ of all deaths in 1994 in China $[2,3]$, and the prevalence of hypertension increased from $11.3 \%$ in 1991 to $27.2 \%$ in 2000 [4,5].

*Corresponding author: Xiao-Ou Shu, M.D., Ph.D., Professor, Department of Medicine, Vanderbilt Epidemiology Center, 2525 West End Avenue, Suite 600, Nashville, TN 37203-1738, USA. Tel.: +1 615936 0713; Fax: +1 615936 8291; E-mail: Xiao-Ou. Shu@vanderbilt.edu.
There is now convincing evidence that elevated blood levels of total cholesterol and low-density lipoprotein (LDL) cholesterol are associated with increased CVD risk, whereas elevated high-density lipoprotein (HDL) cholesterol concentrations appear to provide protection against premature CVD [6-8]. Although lipid biomarkers are often of primary interest in epidemiological studies as they relate to many chronic diseases such as metabolic syndrome and cancer, for economic and logistical reasons it is difficult for such studies to collect either fasting blood samples or multiple blood samples. The degree to which a single sample reflects long-term biomarker levels for an individual depends on the within-person variability of the biomarker over time [9]. The larger the variation, the larger the degree 
of measurement error associated with a single measurement and, as a consequence, observed associations (e.g., relative risk) could be attenuated. Therefore, knowledge of how well a single biomarker measurement reflects longer term levels is important for conducting and interpreting epidemiological studies investigating the associations between those biomarkers and the incidence/prevalence of chronic diseases [10].

We evaluated the reliability and stability of lipid biomarkers and the utility of a single spot blood sample for measuring lipid levels for future large epidemiological studies in a subset of men who participated in the dietary validation of the Shanghai Men's Health Study (SMHS).

\section{Methods}

\subsection{Subjects}

The SMHS is a population-based prospective cohort study with a primary aim to investigate the effect of dietary, occupational, and lifestyle factors and genetic susceptibility on the risk of cancers and other major chronic diseases. Recruitment for the SMHS started in April 2002 and was completed in June 2006. A total of 61,582 men aged 40 to 74 years were recruited into the study, with a participation rate of $74.1 \%$. The SMHS food frequency questionnaire (FFQ) was evaluated in a validation study that included 196 men who were randomly selected from SMHS participants between April 2003 and May 2004 [11]. The validation study included the administration of two FFQs one year apart, 12 monthly 24-hour dietary recalls, and the collection of a blood and urine sample in each quarter of the one-year study period. The overall response rate for the validation study was $69.3 \%$. For the current study, 48 men were randomly chosen from validation study participants who had provided four blood samples throughout the year. Participants of the validation study and parent study were comparable in age, body mass index, waist-to-hip ratio, education, individual income, alcohol consumption, cigarette smoking rates, and total energy intake (data not shown). The study was approved by the Institutional Review Boards of all participating institutions, and all participants provided written informed consent.

\subsection{Estimation of dietary intake and other selected lifestyle factors}

Baseline information including age, physical activity, occupation history, disease history, and dietary habits was collected by an in-person interview. The consumption of each food item or food group was measured with the validated FFQ based on frequency (daily, weekly, monthly, yearly, or never) and amount consumed in liang (one liang $=50 \mathrm{~g}$ ) per unit of time. FFQ items used for estimation of fat intake included fat from animal foods (red meat, poultry, fish, eggs, and milk) and fat from plant foods (vegetables, soy, fruits, and other plant sources). Fatty acids were divided into saturated, monounsaturated, and polyunsaturated fatty acids. Consumption of $\alpha$-linolenic acid (18:3), eicosapentaenoic acid (20:5), and docosahexaenoic acid (22:6) were summed to derive crude omega3 fatty acid intake, and consumption of linoleic acid (18:2), $\gamma$-linolenic acid (20:3), and arachidonic acid (20:4) were summed to derive crude omega-6 fatty acid intake.

Using a standard protocol, trained interviewers measured weight, circumferences of the waist and hips, sitting and standing height, and blood pressure for all participants. The anthropometric measurements were taken twice, and a third measurement was administered if the difference between the first two exceeded the tolerance limits ( $1 \mathrm{~cm}$ for height and $1 \mathrm{~kg}$ for weight). We calculated body mass index (BMI) as weight in kilograms divided by the square of height in meters.

\subsection{Analysis of serum lipids}

Blood samples were collected using EDTA-containing BD Vacutainer ${ }^{\circledR}$ tubes from study participants at the time of their in-person interview without a specific requirement that participants fast beforehand. Blood samples were immediately placed in a cooler with blue ice and processed within 6 hours. Plasma samples were stored at $-70^{\circ} \mathrm{C}$. Among the 48 participants of this reliability study, 30 men provided four non-fasting blood samples; 18 men provided two to three fasting blood samples; and one subject provided four fasting blood samples. Fasting status was defined as an interval between the last meal and blood draw of 8 hours or longer. The levels of selected lipid biomarkers were measured by the Vanderbilt Lipid Laboratory, which is standardized by the Centers for Disease Control and Prevention for lipid analysis, using the $\mathrm{ACE}^{\circledR}$ Clinical Chemistry System (Alfa Wassermann, 
Inc, West Caldwell, NJ). Levels of triglycerides, total cholesterol, and HDL-cholesterol were assayed using the $\mathrm{ACE}^{\circledR}$ Triglyceride Reagent (\#SA1023), ACE ${ }^{\circledR}$ Cholesterol Reagent (\#SA1010), and ACE ${ }^{\circledR}$ HDLC Reagent (\#SA1038) (Alfa Wassermann, Inc, West Caldwell, NJ), respectively, following the manufacturer's protocols. LDL-cholesterol levels were calculated with the Friedwald equation [12] ([LDL-cholesterol] $=[$ Total cholesterol $]-[$ HDL-cholesterol $]-([$ triglycerides]/5)). In subjects with triglyceride levels above $400 \mathrm{mg} / \mathrm{dL}$, LDL-cholesterol levels were measured directly using ACE ${ }^{\circledR}$ LDL-C Reagent (\#SA1040). Coefficient variations (CVs) for intra-batch variation were $2.36,1.24,1.31$, and 3.22 for total cholesterol, triglycerides, HDL-cholesterol, and LDL-cholesterol, respectively. The CVs for inter-batch variation were 3.36, $2.84,2.13$, and 4.65 for total cholesterol, triglycerides, HDL-cholesterol, and LDL-cholesterol, respectively. All four samples for each study participant were measured in the same analytic batch to avoid the influence of batch-to-batch variation.

\subsection{Statistics}

Demographic characteristics and selected lifestyle factors of participants in the validation study were compared with the parent cohort using the Z-test (data not shown). The Student's t-test was employed to compare lipid biomarker levels between fasting and non-fasting blood, and the ANOVA test was used to compare levels across four seasons. To evaluate the stability and usefulness of a single spot blood measurement as an objective biomarker, we evaluated the Spearman correlation between randomly chosen spot blood measurements $(4 * 48=192$ measurements $)$ and the average of the four measurements provided by each subject. The correlation estimates and their $95 \%$ confidence intervals, $(95 \% \mathrm{CI})$ were derived using the bootstrap method with 2,000 repeats in each case. Finally, intraclass correlation coefficients (ICC) were estimated to evaluate the seasonal variability of blood lipid biomarkers using the following one-way random effect model:

$$
\begin{aligned}
& \quad \text { ICC }(\rho)=\sigma_{r}^{2} /\left(\sigma_{r}^{2}+\sigma_{w}^{2}\right) \\
& =\text { [between group variance / (between group vari- } \\
& \text { ance }+ \text { within group variance)] }
\end{aligned}
$$

To evaluate the influence of the observed measurement error in plasma lipid biomarkers on relative risk estimates for future etiological studies, we estimated the relative risk that would be observed given true relative risks of $1.5,2.0$, and 2.5 by multiplying the natural logarithm of the specified true relative risks with the ICC and exponentiated the result [14]. The correlation of lipid biomarkers with dietary fat intake and other lifestyle factors were evaluated using Spearman correlation coefficients adjusting for the time interval between the last meal and blood collection, medicine use, number of cigarettes smoked within the last 24 hours, and total energy intake. All statistical analyses were carried out using SAS, version 9.1 (SAS Institute, Inc, Cary, NC).

\section{Results}

The levels of lipid-related biomarkers from nonfasting blood samples were similar to those from fasting samples with the exception of triglyceride levels (Table 1). The median levels were 177.5, 164.5, 41.0, and $102.5(\mathrm{mg} / \mathrm{dl})$ for total cholesterol, triglycerides, HDL-cholesterol, and LDL-cholesterol, respectively, and levels did not appear to vary greatly by season $(0.10$ $\leqslant \mathrm{p} \leqslant 0.95)$. The correlations between the average intake of these biomarkers across four seasons and during a single season based on bootstrap analysis were also high, ranging from 0.83 (LCL-cholesterol) to 0.93 (HDL-cholesterol) (Table 2).

The ICCs of the measurements across four seasons for total cholesterol, triglycerides, HDL-cholesterol, and LDL-cholesterol were $0.75,0.65,0.83$, and 0.58, respectively (Table 3 ). Given the observed ICC and assuming that the true relative risks of the disease-lipid association were 1.5, 2.0, 2.5, the observed relative risk would be attenuated from $68.0 \%$ to $93.3 \%$ (Table 3 ).

Total cholesterol levels were positively correlated with age $(r=0.30)$. Triglyceride levels were associated with BMI ( $r=0.564)$ and systolic blood pressure $(r=0.33)$. On the other hand, HDL-cholesterol levels were inversely associated with BMI $(r=-0.43)$. No obesity-related markers were associated with LDLcholesterol levels (Table 4).

The patterns of correlation between fat and blood lipid biomarkers differed by fat sources. Total cholesterol was positively associated with fat from red meat $(r=0.15)$ and poultry $(r=0.18)$, but inversely associated with fat from fish $(r=-0.20)$ and soy $(r=$ $-0.27)$. Triglycerides were related to fat from eggs $(r=-0.18)$, milk $(r=0.19)$, and other plant sources $(r=-0.21)$. High HDL-cholesterol levels were associated with high fat intake from fish $(r=0.17)$ and eggs $(r=0.16)$, but was inversely associated with vegetable oil intake $(r=-0.18)$. LDL-cholesterol levels were positively associated with poultry fat $(r=0.24)$, 
Table 1

Comparison of distribution of blood lipid biomarkers between fasting and non-fasting blood samples

\begin{tabular}{|c|c|c|c|c|c|c|c|}
\hline & \multicolumn{3}{|c|}{ Fasting blood sample ${ }^{\mathrm{a}}(n=32)$} & \multicolumn{3}{|c|}{ Non-fasting sample $(n=160)$} & \multirow{2}{*}{$\begin{array}{c}\text { Wilcoxon rank } \\
\text { sum test }\end{array}$} \\
\hline & Mean \pm SD & Median & $(\mathrm{Q} 1, \mathrm{Q} 3)^{\mathrm{b}}$ & Mean \pm SD & Median & $(\mathrm{Q} 1, \mathrm{Q} 3)$ & \\
\hline Cholesterol (mg/dl) & $176.3 \pm 22.4$ & 175.0 & $(161.5,185.5)$ & $180.3 \pm 31.2$ & 178.0 & $(162.5,196.5)$ & 0.39 \\
\hline Triglycerides (mg/dl) & $138.4 \pm 71.1$ & 113.5 & $(94.5,177.0)$ & $218.5 .2 \pm 198.2$ & 169.5 & $(120.0,238.0)$ & 0.01 \\
\hline HDL-cholesterol (mg/dl) & $44.5 \pm 10.2$ & 43.0 & $(36.5,49.5)$ & $41.7 \pm 9.5$ & 40.0 & $(35.0,47.0)$ & 0.15 \\
\hline LDL-cholesterol (mg/dl) & $104.2 \pm 24.2 .3$ & 105.5 & $(89.5,116.0)$ & $99.8 \pm 26.8$ & 102.0 & $(82.5,115.0)$ & 0.29 \\
\hline
\end{tabular}

a"Fasting" refers to blood samples collected 8 hours or more after the most recent meal.

${ }^{\mathrm{b}} \mathrm{Q} 1$ is 25 percentile and $\mathrm{Q} 3$ is 75 percentile.

Table 2

Median levels of blood lipid biomarkers by season and correlation

\begin{tabular}{|c|c|c|c|c|c|c|c|c|c|c|c|}
\hline & \multicolumn{6}{|c|}{ Median (mg/dl) } & \multicolumn{4}{|c|}{ Correlation $(95 \% \mathrm{CI})^{\mathrm{a}}$} & \multirow{2}{*}{$\mathrm{r}(95 \% \mathrm{CI})^{\mathrm{b}}$} \\
\hline & Winter & Spring & Summer & Fall & Average $^{\mathrm{C}}$ & $\mathrm{P}_{\mathrm{F}-\text { test }}$ & Winter & Spring & Summer & Fall & \\
\hline Cholesterol & 179.5 & 181.2 & 175.1 & 177.3 & 177.5 & 0.39 & $\begin{array}{c}0.89 \\
(0.81-0.94)\end{array}$ & $\begin{array}{c}0.91 \\
(0.84-0.95)\end{array}$ & $\begin{array}{c}0.90 \\
(0.83-0.94)\end{array}$ & $\begin{array}{c}0.92 \\
(0.86-0.95)\end{array}$ & $\begin{array}{c}0.91 \\
(0.83-0.95)\end{array}$ \\
\hline Triglycerides & 137.5 & 165.0 & 176.5 & 164.5 & 155.5 & 0.95 & $\begin{array}{c}0.91 \\
(0.84-0.95)\end{array}$ & $\begin{array}{c}0.86 \\
(0.76-0.92)\end{array}$ & $\begin{array}{c}0.82 \\
(0.70-0.90)\end{array}$ & $\begin{array}{c}0.84 \\
(0.73-0.91)\end{array}$ & $\begin{array}{c}0.86 \\
(0.72-0.93)\end{array}$ \\
\hline HDL-cholesterol & 42.0 & 41.0 & 38.5 & 42.5 & 41.0 & 0.34 & $\begin{array}{c}0.94 \\
(0.89-0.97)\end{array}$ & $\begin{array}{c}0.96 \\
(0.93-0.98)\end{array}$ & $\begin{array}{c}0.93 \\
(0.98-0.96)\end{array}$ & $\begin{array}{c}0.95 \\
(0.91-0.97)\end{array}$ & $\begin{array}{c}0.93 \\
(0.89-0.96)\end{array}$ \\
\hline LDL-cholesterol & 106.0 & 101.0 & 96.0 & 89.0 & 102.5 & 0.10 & $\begin{array}{c}0.80 \\
(0.67-0.88)\end{array}$ & $\begin{array}{c}0.82 \\
(0.70-0.90)\end{array}$ & $\begin{array}{c}0.83 \\
(0.71-0.90)\end{array}$ & $\begin{array}{c}0.85 \\
(0.74-0.91)\end{array}$ & $\begin{array}{c}0.83 \\
(0.71-0.92)\end{array}$ \\
\hline
\end{tabular}

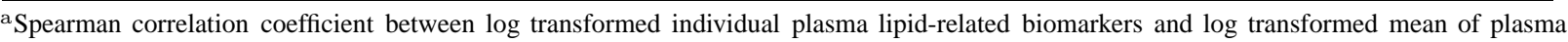
lipid-related biomarkers after adjustment for interval between last meal and blood collection, medicine use, number of cigarettes smoked within the previous 24 hours, and total energy intake.

${ }^{\mathrm{b}}$ Correlations between a randomly chosen individual measurement and averaged measurements, estimated using the bootstrap method.

${ }^{\mathrm{c}}$ Average of four seasonal samples.

but inversely associated with fat from fish $(r=-0.23)$ and soy $(r=-0.28)$. Although total polyunsaturated fatty acids were not associated with lipid biomarkers, omega-3 fatty acid was inversely related to total cholesterol $(r=-0.25)$ and LDL-cholesterol $(r=-0.26)$. Omega-6 fatty acid was positively associated with HDL-cholesterol ( $r=0.18$ ), but inversely associated with LDL-cholesterol $(r=-0.16)$. Total cholesterol intake was positively correlated with HDL-cholesterol level $(r=0.17)$, total triglycerides $(r=0.37)$, and LDL-cholesterol $(r=0.72)$. Triglycerides were negatively correlated with HDL-cholesterol $(r=-0.47)$ (Table 4).

\section{Discussion}

This study found no statistically significant differences in the blood levels of lipid biomarkers between fasting blood and non-fasting blood with the exception of triglycerides. In addition, a single spot blood lipid biomarker measurement correlated well with average levels and could be used to evaluate lipid status in large epidemiological studies.

The use of fasting lipoprotein analysis for the measurement of total cholesterol, triglyceride, HDL- cholesterol, and calculated LDL-cholesterol levels is recommended for clinical diagnosis and monitoring of CVD, diabetes, and hypertension. However, for use in large population-based studies, economic and logistical reasons make the collection of fasting blood samples difficult and impractical. In the present study, we found that the levels of lipid biomarkers obtained from non-fasting blood samples were similar to those obtained from fasting blood. Our finding is consistent with a previous report from a cross-sectional study that showed non-fasting HDL-cholesterol levels were similar to fasting HDL levels, and the agreement in classification of patients into desirable- and high-cholesterol groups using either fasting or non-fasting blood samples was high [15]. LDL-cholesterol and triglyceride levels are more prone to the influence of dietary cholesterol intake. We observed higher within-person variation (lower ICCs) for these two lipid biomarkers than for HDL-cholesterol when test samples were mixed with fasting and non-fasting samples. This suggests that a fasting blood sample may more desirable if LDLcholesterol and triglyceride levels are to be used in epidemiological studies.

In the present study, total cholesterol level was positively correlated with age, and triglyceride levels increased with high BMI and systolic blood pressure, 
Table 3

ICCs for repeated measurements of plasma lipid biomarkers and the resulting observed relative risk for specified true relative risk of 1.5-2.5

\begin{tabular}{lcccc}
\hline Plasma lipid-related biomarker & ICC $^{\mathrm{a}}$ & \multicolumn{3}{c}{ Observed relative risk given specified true relative risk } \\
\cline { 3 - 5 } & & $1.5^{\mathrm{b}}(\%)$ & 2.0 & 2.5 \\
\hline Cholesterol & 0.75 & $1.4^{\mathrm{c}}(93.3)^{\mathrm{d}}$ & $1.7(85.0)$ & $2.0(80.0)$ \\
Triglycerides & 0.65 & $1.3(86.7)$ & $1.6(80.0)$ & $1.8(72.0)$ \\
HDL-cholesterol & 0.83 & $1.4(93.3)$ & $1.8(90.0)$ & $2.1(84.0)$ \\
LDL-cholesterol & 0.58 & $1.3(86.7)$ & $1.5(75.0)$ & $1.7(68.0)$ \\
\hline
\end{tabular}

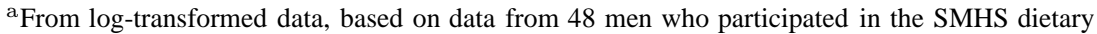
validation study. Levels of selected lipid-related biomarkers are represented as $\mathrm{mg} / \mathrm{dl}$ of plasma. ${ }^{\mathrm{b}}$ True relative risk.

${ }^{\mathrm{c}}$ Observed relative risk.

dPercent of attribution.

Table 4

Correlations* and confidence intervals between blood lipid biomarkers and possible lipid biomarker-related factors

\begin{tabular}{|c|c|c|c|c|}
\hline & Cholesterol & Triglycerides & HDL-cholesterol & LDL-cholesterol \\
\hline Age & $0.30(0.02,0.54)$ & $0.19(-0.10,0.45)$ & $0.09(-0.20,0.36)$ & $0.17(-0.12,0.43)$ \\
\hline Body mass index & $0.24(-0.05,0.49)$ & $0.56(0.33,0.73)$ & $-0.43(-0.64,-0.17)$ & $0.01(-0.27,0.29)$ \\
\hline Waist-to-hip ratio & $0.02(-0.37,0.30)$ & $0.22(-0.07,0.47)$ & $-0.26(-0.51,0.03)$ & $-0.03(-0.31,0.26)$ \\
\hline Systolic blood pressure & $0.23(-0.06,0.48)$ & $0.33(0.06,0.56)$ & $-0.15(-0.42,0.14)$ & $0.05(-0.24,0.33)$ \\
\hline Diastolic blood pressure & $0.11(-0.18,0.38)$ & $0.28(-0.01,0.53)$ & $-0.23(-0.48-0.06)$ & $-0.05(-0.34,0.23)$ \\
\hline Physical activity & $0.05(-0.24,0.33)$ & $-0.01(-0.29,0.27)$ & $-0.01(-0.29,0.27)$ & $0.04(-0.25,0.32)$ \\
\hline Total energy intake & $-0.11(-0.38,0.18)$ & $-0.24(-0.49,0.05)$ & $0.08(-0.21,0.36)$ & $0.10(-0.90,0.37)$ \\
\hline Total Fat & $0.02(-0.27,0.30)$ & $0.07(-0.22,0.35)$ & $-0.12(-0.39,0.17)$ & $0.06(-0.23,0.34)$ \\
\hline \multicolumn{5}{|l|}{ Fat from animal foods } \\
\hline Red meat & $0.15(-0.14,0.42)$ & $-0.03(-0.31,0.26)$ & $-0.08(-0.36,0.21)$ & $0.13(-0.16,0.40)$ \\
\hline Poultry & $0.18(-0.11,0.44)$ & $-0.02(-0.30,0.26)$ & $-0.06(-0.34,0.23)$ & $0.24(-0.05,0.49)$ \\
\hline Fish & $-0.20(-0.46,0.09)$ & $-0.06(-0.34,0.23)$ & $0.17(-0.12,0.43)$ & $-0.23(-0.48,0.06)$ \\
\hline Eggs & $0.04(-0.25,0.32)$ & $-0.18(-0.44,0.11)$ & $0.16(-0.13,0.42)$ & $0.07(-0.22,0.35)$ \\
\hline Milk & $0.11(-0.18,0.38)$ & $0.19(-0.10,0.45)$ & $0.09(-0.20,0.36)$ & $-0.04(-0.32,0.25)$ \\
\hline \multicolumn{5}{|l|}{ Fat from plant foods } \\
\hline Vegetables & $-0.04(-0.32,0.45)$ & $0.05(-0.24,0.33)$ & $-0.18(-0.44,0.11)$ & $0.09(-0.20,0.36)$ \\
\hline Soy & $-0.27(-0.51,0.02)$ & $0.12(0.17,0.39)$ & $-0.07(-0.34,0.22)$ & $-0.28(-0.53,0.00)$ \\
\hline Fruits & $-0.01(-0.29,0.27)$ & $-0.06(-0.34,0.23)$ & $-0.01(-0.29,0.27)$ & $0.01(-0.27,0.29)$ \\
\hline Other & $-0.06(-0.34,0.23)$ & $-0.21(-0.47,0.08)$ & $0.12(-0.17,0.39)$ & $0.06(-0.23,0.34)$ \\
\hline Saturated FA & $0.14(-0.15,0.41)$ & $0.02(-0.27,0.30)$ & $0.01(-0.27,0.29)$ & $0.09(-0.20,0.37)$ \\
\hline Monounsaturated FA & $0.11(-0.18,0.38)$ & $-0.02(-0.30,0.27)$ & $0.01(-0.27,0.29)$ & $0.11(-0.18,0.38)$ \\
\hline Polyunsaturated FA & $-0.08(-0.36,0.21)$ & $-0.01(-0.29,0.27)$ & $0.05(-0.24,0.33)$ & $-0.06(-0.34,0.23)$ \\
\hline$\omega-3$ fatty acids & $-0.25(-0.50,0.04)$ & $0.02(-0.27,0.30)$ & $0.04(-0.25,0.32)$ & $-0.26(-0.51,0.03)$ \\
\hline$\omega-6$ fatty acids & $-0.15(-0.42,0.14)$ & $-0.07(-0.35,0.22)$ & $0.18(-0.11,0.44)$ & $-0.16(-0.42,0.13)$ \\
\hline Dietary cholesterol & $0.01(-0.27,0.29)$ & $-0.12(-0.39,0.17)$ & $0.17(-0.12,0.43)$ & $0.01(-0.27,0.29)$ \\
\hline \multicolumn{5}{|l|}{ Blood lipid biomarkers } \\
\hline Total cholesterol & 1.00 & & & \\
\hline Triglycerides & $0.37(0.10,0.59)$ & 1.00 & & \\
\hline HDL-cholesterol & $0.21(-0.08,0.47)$ & $-0.47(-0.67,-0.21)$ & 1.00 & \\
\hline LDL-cholesterol & $0.72(0.55-0.83)$ & $-0.18(-0.44,0.11)$ & $0.24(-0.05,0.49)$ & 1.00 \\
\hline
\end{tabular}

${ }^{*}$ Spearman correlation coefficient after adjustment for interval between last meal and blood collection, medicine use, number of cigarettes smoked within the previous 24 hours, and total energy intake. Dietary information, blood pressure and anthropometrics were based on baseline survey data.

Bold indicates statistical significance $(p<0.05)$ for Spearman correlation coefficient analyses.

while HDL-cholesterol was inversely associated with BMI similar to other previous reports [16]. The correlation between dietary fat intake and blood lipid biomarkers varied according to the fat source in this study. Blood lipids and lipoproteins have been shown to be associated with dietary fat intake. Several studies have indicated that low-fat and high-carbohydrate diets re- duce both LDL-cholesterol and HDL-cholesterol [17$20]$, and may also raise triglycerides [17,19], an independent risk factor for the development of CHD [21]. In our study, levels of total and LDL-cholesterol were inversely correlated with intake of omega-3 and omega6 fatty acids, although total saturated or unsaturated fatty acids were not associated with any lipid biomark- 
ers. The absolute concentration of LDL-cholesterol achieved in humans [22] or hamsters [23] has previously been shown to be positively associated with a diet predominated by saturated fatty acids but inversely associated with a diet predominated by unsaturated fatty acids. It should be noted that the number of subjects included in the current study is small, and estimates of the associations between dietary fatty acids and plasma lipid biomarker levels were not stable. Further studies on this topic are needed.

It is a common practice in epidemiological studies to classify individuals into different groups according to their exposure levels. If the within-person variability of biomarker levels is random, then the correlation of a single measurement with the average of multiple measurements in a population can be used to 'correct' the attenuation of relative risk estimates by using a single measurement [9]. In our study, all lipid-related biomarkers were shown to have reasonably high ICC values, suggesting small within-person variability and large between-person variability. An ICC of 0.65 or larger results in relatively modest decreases in the estimated relative risk, although the degree of attenuation that can be tolerated depends on the size of the study and the magnitude of the risk [14]. Correction for measurement error is particularly important in the case of exposure measurements, such as LDL-cholesterol ( ICC $=0.58)$, where the level of error can result in appreciable attenuation of relative risk estimates.

In conclusion, this study suggests that levels of lipid biomarkers from a single spot blood sample are relatively stable in this cohort of Chinese men. Thus, a single spot blood sample can serve as a useful resource for future studies of associations between lipid levels and health.

\section{Acknowlegment}

We thank Dr. Qing Wang and Ms. Kimberly Campbell for their contributions in processing and measuring biological samples and Ms. Bethanie Hull for her assistance in the preparation of this manuscript. This study would not have been possible without the support of the study participants and research staff of the Shanghai Men's Health Study. This work was supported by USPHS grant R01CA82729 from the National Cancer Institute.

\section{References}

[1] D. Gu, K. Reynold, X. Wu, J. Chen, X. Duan, R. Reynold, P.K. Whelton and J. He, for the InterASIA Collaborative Group. Prevalence of the metabolic syndrome and overweight among adults in China, Lancet 365 (2005), 1398-1405.

[2] C.J.L. Murray and A.D. Lopez, The Global Burden of Disease: a comprehensive assessment of mortality and disability from diseases, injuries, and risk factors in 1990 and projected to 2020, $1^{\text {st }}$ ed. Cambridge, Mass: Harvard University Press; 1996.

[3] World Health Organization. World Health Statistics Annual. Geneva, Switzerland: World Health Organization; 1996.

[4] X. Wu, X. Duan, D. Gu, J. Hao, S. Tao and D. Fan, Prevalence of hypertension and its trends in Chinese populations, Int $J$ Cardiol 52 (1995), 39-44.

[5] D. Gu, K. Reynolds, X. Wu, J. Chen, X. Duan, P. Muntner, G. Huamg, R.F. Reynolds, S. Su, P.K. Whelton and J. He, Prevalence, awareness, treatment, and control of hypertension in China, Hypertension 40 (2002), 920-927.

[6] D.J. Gordon, J.L. Probstfield, R.J. Garrison, J.D. Neaton, W.P. Vastelli, J.D. Knoke, D.R. Jacob Jr., Bangdiwala S, Tyroler HA. High density lipoprotein cholesterol and cardiovascular disease: Four prospective American studies, Circulation 79 (1989), 8-15.

[7] Lipid Research Clinics Program. The Lipid Research Clinics Coronary Primary Prevention Trial results. II. The relationship of reduction in incidence of coronary heart disease to cholesterol lowering, JAMA 251 (1984), 365-374.

[8] W.P. Castelli, R.J. Garrison, P.W.F. Wilson, R.D. Abbott, S. Kalousdian and W.B. Kannel, Incidence coronary heart disease and lipoprotein cholesterol levels: The Framingham Study, JAMA 256 (1986), 2835-2838.

[9] D.J. Hunter, Methodological issues in the use of biological markers in cancer epidemiology: cohort studies, in: Application of biomarkers in cancer epidemiology, P. Toniolo, P. Boffetta, D.E.G. Shuker, N. Rothman, B. Hulka and N. Pearce, eds, IARC Scientific Publication No. 142, International Agency for Research on Cancer, Lyon, 1997, pp. 39-46.

[10] S.E. Hankinson, J.E. Manson, D. Spiegelman, W.C. Willett, C. Longcope and F.E. Speizer, Reproducibility of plasma hormone levels in postmenopausal women over 2-3-year period, Cancer Epidemiol Biomarkers Prev 4 (1995), 649-654.

[11] R. Villegas, G. Yang, D. Liu, Y.-B. Xiang, H. Cai, W. Zheng and X.O. Shu, Validation and reproducibility of the food frequency questionnaire used in the Shanghai Men's Health Study, Br J Nutr 7 (2007), 1-8.

[12] W.T. Friedwald, R.L. Levy and D.S. Fredrickson, Estimation of the concentration of low density lipoprotein cholesterol in plasma without the use of preparative ultracentrifuge, Clin Chem 18 (1972), 499-502.

[13] K.O. Mc Graw, Forming inferences about some interclass correlation coefficients, Phychological Methods 1(1) (1996), 3046.

[14] B. Rosner, D. Spiegelman and W.C. Willett, Correction of logistic regression relative risk estimates and confidence intervals for random within-person measurement error, $\mathrm{Am} \mathrm{J}$ Epidemiol 136 (1992), 1400-1413.

[15] S.R. Craig, R.V. Amin, D.W. Russell and N.F. Paradiase, Blood cholesterol screening: influence of fasting state on cholesterol results and management decisions, J Gen Intern Med 15 (2000), 395-399. 
[16] C.S. Yajnik, The lifecycle effects of nutrition and body size on adult adiposity, diabetes, and cardiovascular disease, Obes $\operatorname{Rev} 3$ (2002), 217-224.

[17] S.E. Kasim-Karakas, R.U. Almario, W.M. Mueller and J. Peerson, Changes in plasma lipoproteins during low-fat, highcarbohydrate diets: effects of energy intake, Am J Clin Nutr 71 (2000), 1439-1447.

[18] E.J. Parks, R.M. Krauss, M.P. Christiansen, R.A. Neese and M.K. Hellerstein, Effects of a low-fat, high-carbohydrate diet on VLDL-triglyceride assembly, production, and clearance, $J$ Clin Invest 104 (1999), 1087-1096.

[19] D. Ullmann, W.E. Connor, L.F. Hatcher, S.L. Connor and D.P. Flavell, Will a high-carbohydrate, low-fat diet plasma lipids and lipoproteins without producing hypertriglyceridemia? $\mathrm{Ar}$ terioscler Thromb 11 (1991), 1059-1067.

[20] H. Muller, A.S. Lindman, A.L. Brantsaeter and J.I. Pedersen,
The serum LDL/HDL cholesterol ratio is influenced more favorably by exchanging saturated with unsaturated fat than by reducing saturated fat in the diet of women, J Nutr $\mathbf{1 3 3}$ (2003), $78-83$

[21] M.A. Austin, J.E. Hokanson and K.L. Edwards, Hypertriglyceridemia as a cardiovascular risk factor, Am J Cardiol $\mathbf{8 1}$ (1998), 7B-12B.

[22] C.J. Fielding, R.J. Havel, K.M. Todd, K.E. Yeo, M.C. Schloetter and V. Weinberg, Effects of dietary cholesterol and fat saturation on plasma lipoproteins in an ethnically diverse population of healthy young men, J Clin Invest 95(2) (1995), 611-618.

[23] D.K. Spady and J.M. Dietschy, Interaction of dietary cholesterol and triglycerides in the regulation of hepatic low density lipoprotein transport in the hamster, J Clin Invest 81 (1988), 300-309. 


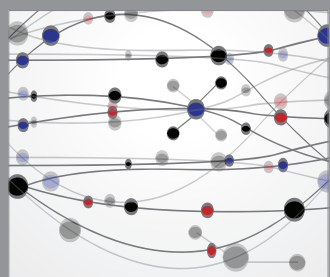

The Scientific World Journal
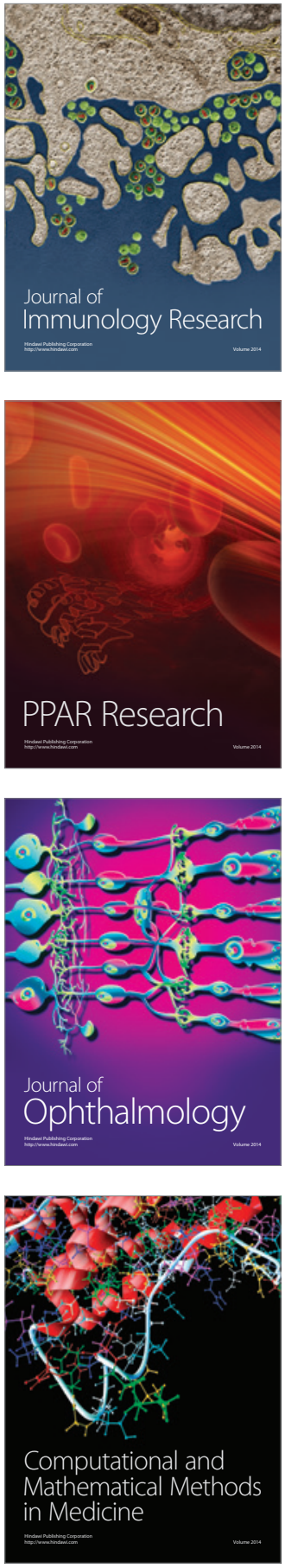

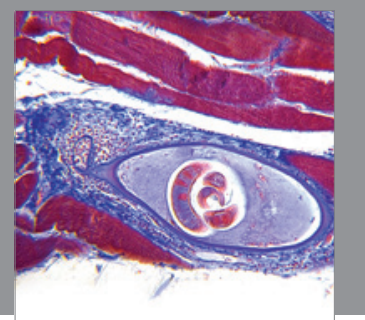

Gastroenterology

Research and Practice
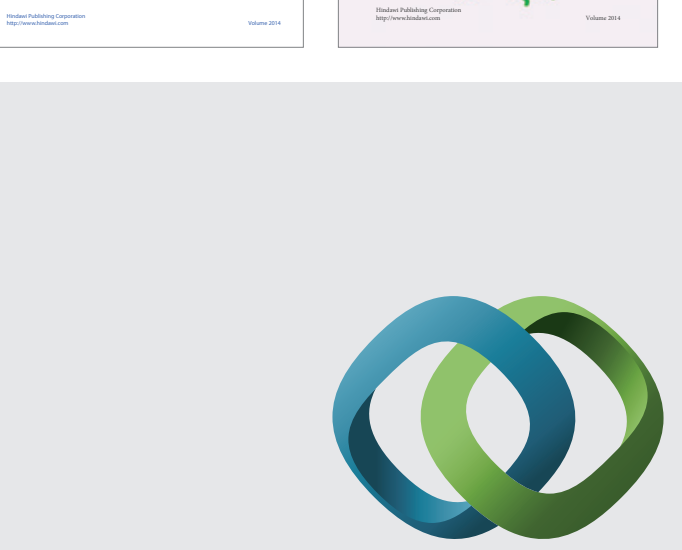

\section{Hindawi}

Submit your manuscripts at

http://www.hindawi.com
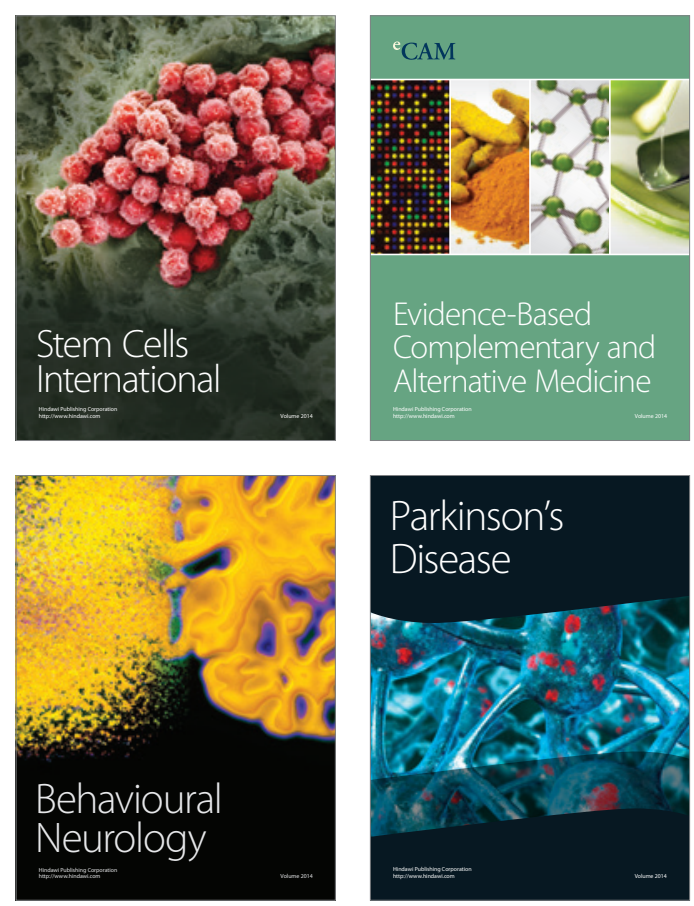

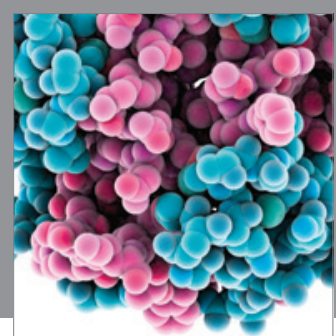

Journal of
Diabetes Research

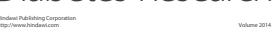

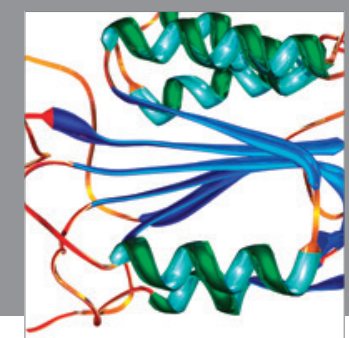

Disease Markers
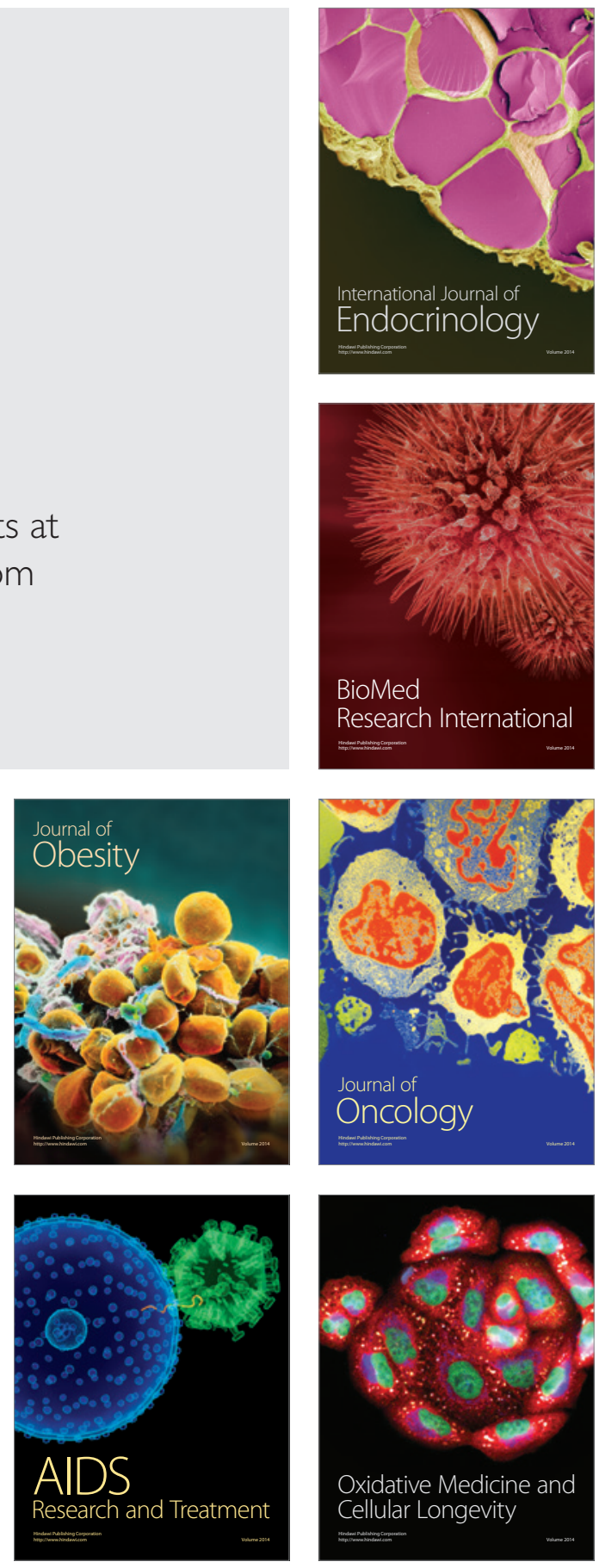